Service social

\title{
Un groupe de parents d'adolescents : son cheminement, de l'éducation structurée au modèle à buts sociaux
}

\section{Camile Spain}

Volume 35, numéro 3, 1986

Les jeunes et le travail social

URI : https://id.erudit.org/iderudit/706318ar

DOI : https://doi.org/10.7202/706318ar

Aller au sommaire du numéro

Éditeur(s)

École de service social de l'Université Laval

ISSN

1708-1734 (numérique)

Découvrir la revue

Citer cet article

Spain, C. (1986). Un groupe de parents d'adolescents : son cheminement, de l'éducation structurée au modèle à buts sociaux. Service social, 35(3), 352-365. https://doi.org/10.7202/706318ar
Résumé de l'article

Le cheminement de parents d'adolescents ayant participé à une session offerte par le C.L.S.C. Pierrefonds est retracé, et nous est exposé à l'aide de la théorie du service social des groupes.

À l'origine un groupe d'éducation structurée, dont les objectifs étaient l'amélioration des connaissances des phénomènes de l'adolescence et le développement d'habiletés de communication, des parents décident de se donner davantage de support et forment un groupe de développement fonctionnant selon un modèle de réciprocité. Peu à peu celui-ci se transforme en groupe de changement social selon un modèle dit « à buts sociaux ". 


\section{Un groupe de parents d'adolescents : son cheminement, de l'éducation structurée au modèle à buts sociaux}

\section{Camile Spain}

Lors de l'année internationale de la jeunesse, en 1985, des parents organisaient, à Pierrefonds, une journée spéciale consacrée aux parents d'adolescents. Sans vouloir détourner l'attention portée aux jeunes, ils voulaient souligner leur travail et leurs efforts, eux qui se sentent souvent déroutés devant les manifestations de l'adolescence.

Un cheminement de groupe de plus de trois années les a menés à réaliser cette action dans leur communauté. Cet article analysera l'expérience, à l'aide de la théorie du service social des groupes. Pour bien situer le lecteur, nous reprendrons, dans la première partie, leur cheminement général; nous examinerons ensuite le contexte dans lequel le groupe a pris naissance, soit une session pour parents d'adolescents, offerte par le Centre local de services communautaires (C.L.S.C.) Pierrefonds. Nous nous arrêterons enfin aux phases I et II du Club de parents d'adolescents.

\section{Cadre théorique}

Le service social des groupes s'affirme de plus en plus comme une méthode en soi. Darveau-Fournier et Home (1980) en dégagent le caractère spécifique. Leur recherche sur la pratique de cette méthode à Québec (1983 : 134) permet de distinguer, à partir de leurs objectifs, quatre types de groupes : ceux qui visent le changement personnel, ceux qui sont axés à la fois sur le développement des membres et celui de leur milieu, ceux qui sont orientés vers le changement social dans l'environnement et, finalement, ceux qui portent sur la gestion des 
services. C'est à partir de cette typologie que nous classifierons chacune des phases du cheminement du groupe analysé.

Pour préciser davantage le mode de fonctionnement des parents au cours de chacune des périodes, nous utiliserons les distinctions entre les trois modèles du service social des groupes (Papell et Rothman, 1966), soit les modèles de réadaptation, de réciprocité et à buts sociaux. Un article de Papell et Rothman (1983) met en lumière ce qui est commun à ces trois modèles - qu'elles ont appelé le courant central en service social - en parallèle avec les approches extérieures au service social tels le groupe structuré et la psychothérapie. II nous sera particulièrement utile pour parler de la phase de groupe d'éducation structurée. Nous lui emprunterons les quatre éléments, soit les caractéristiques, les membres, les activités et le rôle de l'intervenante, non pour dégager les similitudes, mais plutôt pour faire ressortir les particularités propres à chaque phase. Pour compléter le tableau, nous accorderons une attention particulière à la dimension de l'autonomie du groupe.

\section{Le cheminement général du groupe}

Au printemps 1980, deux intervenantes du C.L.S.C. Pierrefonds élaboraient un programme pour aider les parents d'adolescents dans leur rôle. C'est à partir des consultations, individuelles ou en petits groupes, qu'elles avaient identifié leur besoin d'améliorer leur connaissance et leur compréhension des différentes facettes de l'adolescence ainsi que leur désir de se rapprocher des jeunes. Ce programme s'inspirait d'un projet du Bureau de consultation jeunesse inc. ${ }^{1}$ et prévoyait dix rencontres de deux heures et demie chacune. Les thèmes abordés concernaient les valeurs des jeunes et celles des parents, la psychologie de l'adolescence, la communication, la drogue, la sexualité, les loisirs, etc.

Ce programme existe toujours au C.L.S.C., et plusieurs sessions sont offertes chaque année à des parents aux prises avec les difficultés courantes des manifestations de l'adolescence. Ces rencontres visent l'amélioration des connaissances des phénomènes de l'adolescence et le développement des habiletés de communication. Elles constituent vraiment des groupes structurés où l'on trouve une situation précise d'apprentissage et un programme planifié pour atteindre les objectifs (Drum et Knott, 1977). On y recherche les changements individuels.

C'est à partir des individus ayant participé à la session de l'hiver 1981-1982 que le groupe de parents s'est formé. Cette session avait été 
particulière car les gens y démontraient un plus grand engagement personnel et l'intervenante laissait plus de place aux interactions d'aide. À la fin de la série de rencontres, neuf des treize parents manifestaient le désir de se revoir pour parler des progrès de la relation avec leurs jeunes. Le groupe avait évolué et en était devenu un de référence, ce qui se produit rarement dans un groupe structuré pur (Papell et Rothman, 1983).

En juin 1982, sept parents se sont donc réunis avec l'intervenante chez un des couples participants. Après s'être entretenus, de manière informelle, ils ont clarifié leur demande : que le C.L.S.C. organise des rencontres pour répondre à leur besoin de support dans leur rôle d'éducateur.

Le C.L.S.C. approuvait le travail auprès de ce groupe, sous réserve qu'il devienne rapidement autonome et qu'il puisse éventuellement agir dans le milieu pour améliorer la condition des jeunes, par exemple en exerçant des pressions pour changer les règlements municipaux restreignant l'accès aux parcs. Cependant, le travail fut interrompu avant que le contrat ne soit réellement passé entre le groupe et le C.L.S.C. Malgré une absence prolongée de l'intervenante, les parents se sont tout de même rencontrés à trois reprises durant l'année 1982-1983 chez la personne que nous avions identifiée comme leader potentiel. Insatisfaits de leurs rencontres qu'ils associaient à du verbiage, ils réclamaient plus de "nourriture».

À l'automne 1983, le noyau initial n'étant plus que de cinq parents, qui réitéraient toutefois leur demande au C.L.S.C., il fallait recruter de nouveaux membres pour que l'expérience soit valable. Deux critères de sélection étaient imposés: d'une part, les personnes intéressées devaient avoir déjà suivi une session pour parents d'adolescents au C.L.S.C. ; ceci était une exigence des cinq membres initiaux qui souhaitaient que tous aient amorcé une démarche similaire; d'autre part, ils devaient habiter à proximité les uns des autres afin de pouvoir éventuellement agir sur leur milieu; cette condition était fixée par le C.L.S.C. Plus d'une cinquantaine de personnes ont été invitées et, finalement, cinq autres parents se sont joints à ce noyau initial pour former le Club de parents d'adolescents.

Il était entendu que l'intervenante n'entreprendrait pas une nouvelle session, mais aiderait les parents à aborder les questions qui les intéressaient. La planification du programme et l'organisation des soirées thématiques leur étaient laissées.

La phase I du Club s'est déroulée au cours de l'année 1983-1984. Les objectifs étaient à ce moment axés sur le développement des individus, 
mais aussi sur celui du groupe, de ses ressources et de son autodétermination (Darveau-Fournier et Home, 1980). Les membres ont organisé douze rencontres en huit mois et ont abordé tous les sujets qu'ils avaient sélectionnés au départ. La cohésion s'était fortement développée et l'aide mutuelle se manifestait, car les personnes avaient besoin les unes des autres et collaboraient à résoudre leurs problèmes communs (Shulman, 1984).

À l'automne 1984, le Club amorçait la phase II. Après s'être donnés beaucoup de support mutuel et s'être suffisamment alimentés sur les plans théorique et pratique, les membres décidaient de communiquer leurs acquis aux autres parents du territoire. Durant l'année 1984-1985, ils visaient une action dans la communauté et le changement social devenait leur objectif. Pour l'atteindre, ils ont organisé, en mai 1985, une journée spéciale pour leurs pairs. Cette journée a été un succès : soixante parents, dont vingt-quatre pères, y ont participé. Le groupe, très satisfait de son travail, était enchanté de la réponse reçue. Cependant, il s'est dissout.

À l'automne 1985, le leader du début, devenu responsable officiel au cours de la dernière phase du Club, et son conjoint, décidaient de devenir des ressources pour les parents de la communauté. Ils s'orientaient ainsi vers un groupe d'entraide.

Après avoir décrit, dans ses grandes lignes, le cheminement du groupe, nous allons reprendre plus en détail chacune de ses phases. Le lecteur pourra se référer, au besoin, au tableau 1.

\section{La session pour parents d'adolescents : un groupe structuré d'éducation}

\section{Le groupe, ses caractéristiques}

Comme nous l'avons mentionné, la session, dont les buts étaient prédéterminés, visait l'amélioration des connaissances du phénomène de l'adolescence et le développement des habiletés de communication. Une fois acquises, ces habiletés peuvent être transférées dans d'autres sphères que la relation parent/adolescent (Drum et Knott, 1977). Cela a été le cas pour des personnes qui ont amélioré la communication avec leur conjoint et, entre autres, pour un homme qui a utilisé ses habiletés d'écoute dans une relation d'affaires afin de mieux saisir les besoins et les craintes du client. C'est un bel exemple du groupe d'éducation structurée. 


\section{TABLEAU 1}

\section{Synthèse du cheminement d'un groupe de parents d'adolescents.}

\begin{tabular}{|c|c|c|c|}
\hline & \multirow{2}{*}{ Session pour parents d'adolescents } & \multicolumn{2}{|c|}{ Club de parents d'adolescents } \\
\hline & & Phase I & Phase II \\
\hline Période & Hiver 1982 & Automne 1983-été $1984 *$ & Automne 1984-été 1985 \\
\hline $\begin{array}{l}\text { Type de } \\
\text { groupe }\end{array}$ & $\begin{array}{l}\text { Groupe de changement personnel } \\
\text { (10 parents) }\end{array}$ & $\begin{array}{l}\text { Groupe de développement } \\
\text { ( } 5 \text { parents }+5 \text { nouveaux) }\end{array}$ & $\begin{array}{l}\text { Groupe de changement social } \\
\text { (8 parents) }\end{array}$ \\
\hline $\begin{array}{l}\text { Modèle/ } \\
\text { approche }\end{array}$ & $\begin{array}{l}\text { Approche d'éducation structurée } \\
\text { (extérieur au courant central en service } \\
\text { social) }\end{array}$ & $\begin{array}{l}\text { Modèle de réciprocité } \\
\text { (service social des groupes) }\end{array}$ & $\begin{array}{l}\text { Modèle à buts sociaux } \\
\text { (service social des groupes) }\end{array}$ \\
\hline Objectifs & $\begin{array}{l}\text { Prédéterminés par le C.L.S.C. : } \\
\text { - améliorer les connaissances des diverses } \\
\text { facettes de l'adolescence ; } \\
\text { - développer les habiletés à communi- } \\
\text { quer; } \\
\text { - favoriser le rapprochement parent/ } \\
\text { jeune }\end{array}$ & $\begin{array}{l}\text { Négociés entre groupe-intervenante- } \\
\text { C.L.S.C.: } \\
\text { - approfondir les connaissances des di- } \\
\text { verses facettes de l'adolescence ; } \\
\text { - exercer les habiletés à communiquer }\end{array}$ & $\begin{array}{l}\text { Décidés par le groupe avec l'interve- } \\
\text { nante: } \\
\text { - faire partager aux pairs les acquis ; } \\
\text { - valoriser le rôle de parents d'adolescents } \\
\text { dans la communauté ; } \\
\text { - se donner du support dans le rôle de } \\
\text { parent }\end{array}$ \\
\hline Extériorité & Absente & $\begin{array}{l}\text { Commence à émerger } \\
\text { Des parents du groupe vont expliquer } \\
\text { leur démarche à d'autres parents qui } \\
\text { veulent partir un club }\end{array}$ & $\begin{array}{l}\text { Présente } \\
\text { Le groupe est ouvert à la communauté }\end{array}$ \\
\hline
\end{tabular}




\begin{tabular}{|c|c|c|c|}
\hline Activités & $\begin{array}{l}\text { Planifiées, préparées et animées par } \\
\text { l'intervenante : } \\
\text { exposés, information technique, dis- } \\
\text { cussions, exercices, jeux de rôles, vi- } \\
\text { sionnements, devoirs avec le jeune à la } \\
\text { maison } \\
\text { Sujets abordés : valeurs, psychologie } \\
\text { del'adolescence, communication, sexua- } \\
\text { lité, drogues, loisirs, etc. }\end{array}$ & $\begin{array}{l}\text { Planifiées par le groupe et préparées } \\
\text { à tour de rôle, avecl'aide del'interve- } \\
\text { nante au besoin : } \\
\text { personne ressource invitée, vision- } \\
\text { nements, discussions,échanges surle } \\
\text { vécu, exercices, sorties de groupe, } \\
\text { etc. } \\
\text { Sujets abordés: "nous " face à l'édu- } \\
\text { cation sexuelle, les tâches domestiques, } \\
\text { le désintérêt scolaire }\end{array}$ & $\begin{array}{l}\text { Planifiées par le groupe et partagées } \\
\text { entre les membres selon les aptitudes: } \\
\text { démarches pour trouver des sources de } \\
\text { financement, organisation du programme, } \\
\text { rédaction de communiqués de presse, } \\
\text { organisation du buffet, de la salle, etc. }\end{array}$ \\
\hline $\begin{array}{l}\text { Rôle de } \\
\text { P'intervenante }\end{array}$ & $\begin{array}{l}\text { Enseignante (rôle principal) } \\
\text { Facilitateur d'aide mutuelle entre les } \\
\text { membres (rôle secondaire) }\end{array}$ & $\begin{array}{l}\text { Facilitateur d'aide mutuelle (rôle prin- } \\
\text { cipal) } \\
\text { Enseignante et médiateur } \\
\text { Support à la responsable du groupe. } \\
\text { (rôles occasionnels) }\end{array}$ & $\begin{array}{l}\text { Personne ressource (rôle principal) } \\
\text { Médiateur (rôle occasionnel) }\end{array}$ \\
\hline $\begin{array}{l}\text { Position de } \\
\text { P'intervenante }\end{array}$ & Très centrale & Périphérique & Périphérique \\
\hline Autonomie & Très faible & Groupe semi-autonome & Groupe autonome \\
\hline
\end{tabular}

* Automne 1982-automne 1983 : période de flottement; des rencontres informelles conduisent à la formation du Club de parents d'adolescents. 
L'objectif était le changement personnel, non le traitement, mais par un processus d'apprentissage et par l'identification des sentiments favorisant ou empêchant l'acquisition de la compétence désirée (Papell et Rothman, 1983: 26). Le programme de la session en témoigne. Deux éléments du courant central en service social étaient aussi présents lors de cette session : l'aide mutuelle a été favorisée et une légère attention a été portée au développement du groupe (Papell et Rothman, 1983). Malgré cela, c'est l'approche d'éducation structurée qui était dominante.

Un autre aspect manifeste de l'éducation structurée est la prévention secondaire (avant que le problème ne se manifeste dans la relation). Drum et Knott (1977) identifient trois catégories de groupes structurés : ceux qui visent l'acquisition d'habiletés, ceux qui aident les gens à résoudre des problèmes, comme la solitude par exemple, et ceux qui aident les gens à traverser les étapes de transition, tel le veuvage. C'est à la première catégorie, le groupe de développement des habiletés quotidiennes, que réfère la session des parents d'adolescents parce qu'elle est axée sur l'acquisition d'habiletés favorables aux interrelations entre parents et adolescents.

Contrairement à ce qui se passe habituellement dans les groupes d'éducation structurée, l'autonomie se développait. L'intervenante laissait place aux interactions, notait les besoins qui étaient manifestés et permettait la modification de certains aspects du programme. C'est donc l'émergence d'un principe du service social des groupes qu'on pouvait observer (Darveau-Fournier et Home, 1980).

\section{Les membres}

Nous nous adressions à l'individu dans son rôle de parent qui désire acquérir des compétences dans sa relation avec son adolescent et corriger certaines façons d'agir (Papell et Rothman, 1983). Le groupe d'éducation structurée demande une présélection minimale : dans le cas présent, il fallait avoir des adolescents, souscrire aux objectifs de la session et ne pas venir pour résoudre des problèmes personnels graves.

\section{Les activités}

Les activités du groupe d'éducation structurée sont très diversifiées et choisies en fonction des objectifs. Ainsi, il pouvait y avoir des exposés théoriques (la psychologie de l'adolescence), un apport d'informations techniques (les drogues et leurs effets), des discussions (pourquoi 
certains jeunes abusent-ils des drogues?), des jeux de rôles ou des mises en situation (que dirais-tu à ton fils si tu trouvais du haschich dans sa chambre?), des devoirs à faire avec les jeunes à la maison et des retours sur ces devoirs, etc.

Comme le mentionnent Papell et Rothman (1983), ces activités doivent avoir un ordre précis pour que les apprentissages se fassent au bon moment. Par exemple, le thème portant sur les habiletés à communiquer ses sentiments était abordé à la troisième rencontre, de sorte que les parents puissent les mettre en pratique tout au long des sept autres rencontres. Ce programme rigide ou très structuré diffère de celui du courant central qui émerge plutôt des intérêts et des désirs spontanés des membres, que l'on essaie de satisfaire dans un processus de planification et de collaboration (Papell et Rothman, 1983:17). Ici, la planification a été entièrement assumée par l'intervenante qui a tenu compte, à quelques occasions, des désirs exprimés par les personnes.

\section{Le rôle de l'intervenante}

Le rôle prédominant joué par l'intervenante dans la session offerte aux parents est celui d'enseignante ; elle apportait les éléments théoriques et illustrait les habiletés de communication. Elle a aussi joué le rôle de facilitateur, en supportant l'expression des compétences ou des habiletés et en créant des occasions pour permettre l'exercice de ces habiletés (Germain et Gitterman, 1980). En ce qui concerne l'aide mutuelle, son rôle de facilitateur a été très secondaire, mais en progression constante. L'intervenante occupait donc une position très centrale ; elle se montrait directive et assurait entièrement le leadership. Cela se faisait tout de même dans un climat chaleureux et de confiance. Plus l'intervenante devenait expérimentée, plus elle réduisait la distance la séparant des membres.

Le déroulement de cette session a eu une influence sur les sessions subséquentes qui ont emprunté de plus en plus d'éléments au service social des groupes. Le rôle de l'intervenante s'est transformé, le programme s'est assoupli et l'aide mutuelle a été favorisée. 


\section{Le club de parents d'adolescents, phase I : le modèle de réciprocité}

\section{Le groupe, ses caractéristiques}

Durant l'année 1983-1984, qui correspond à la phase 1 du Club de parents d'adolescents et d'adolescentes, dix parents se sont organisés, avec l'aide du C.L.S.C., pour répondre à leurs besoins. Ce groupe avait le caractère préventif, éducatif et communautaire du service social des groupes (Darveau-Fournier et Home, 1980). En effet, il s'adressait à des parents sans problèmes majeurs, désireux de parfaire leurs connaissances et d'avoir du support face à leur rôle. De plus, il était susceptible de s'ouvrir éventuellement sur la communauté, par exemple, en appuyant la Maison des jeunes.

Dans ce groupe, les membres, comme individus, sont la cible du changement : ils tentent de s'améliorer dans leur rôle et ils recherchent des changements personnels par la compréhension de leurs sentiments, de leurs attitudes ou par l'exercice d'un nouveau comportement. Cependant, quand l'un d'entre eux devient animateur ou organisateur de la soirée, c'est son propre développement, comme personne, qui s'effectue. Il ou elle assume de nouveaux rôles, de nouvelles responsabilités et développe de nouvelles capacités. Dans ce processus, tous gagnent de l'autonomie ; c'est pourquoi nous l'associons au groupe de développement, selon la typologie de Darveau-Fournier et Home (1983).

Même si les objectifs du Club, dans sa phase I, ressemblent à ceux que l'on retrouvait dans le groupe d'éducation structurée, il appartient davantage au courant central, car les trois caractéristiques données par Papell et Rothman (1983: 14) sont présentes : un objectif commun, l'aide mutuelle et les expériences variées. En effet, les objectifs communs des membres ont été négociés lors du contrat entre les différents partenaires (les membres, l'intervenante et le C.L.S.C.) afin de tenir compte des besoins des premiers et des exigences de l'institution. Ce type de contrat suggère le modèle de réciprocité. La deuxième caractéristique du courant central est la présence de l'aide mutuelle, qui s'est très fortement manifestée dans cette première phase. Les parents se donnaient beaucoup de support et leur action les aidait eux-mêmes à prendre confiance en eux et à clarifier leurs positions. Les exemples suivants font ressortir davantage l'aspect de réciprocité dans le processus : l'échange d'expériences où un parent a exprimé les moyens utilisés avec son fils face à son désintérêt scolaire ; la discussion de sujets tabous où les parents ont abordé leur propre sexualité; le phénomène de 
"tout le monde dans le même bateau", en particulier lorsqu'une femme très isolée a pu puiser un certain réconfort dans le groupe et se sentir moins seule dans sa situation; la résolution d'un problème individuel, lorsque tout le groupe a tenté d'aider une mère à faire participer ses enfants et son mari aux tâches domestiques, lui faisant même pratiquer la façon de présenter la demande à ses enfants. Quant à la troisième caractéristique : les expériences variées, nous y reviendrons dans la partie traitant des activités.

Bien que le groupe ait été fermé, l'extériorité, concept clé du courant central, était présente (Papell et Rothman, 1983). Par exemple, des parents sont allés parler de leur expérience de groupe à d'autres parents désireux de former un club; ils ont également appuyé la Maison des jeunes en participant aux préparatifs de l'ouverture officielle. Cependant, le lien avec le milieu n'était pas formel. Ainsi, c'est à titre personnel que deux mères du Club ont été élues au conseil d'administration de la Maison des jeunes.

Le groupe, selon le modèle de réciprocité, se définit par l'alliance d'individus qui ont besoin les uns des autres pour travailler à leurs problèmes communs. C'est la mutualité dont parle Shulman (1984). Selon ce modèle, on travaille sur l'interaction individu/groupe/milieu. C'est ce qui a été fait, mais sans être conscient de tout ce que ce rôle exige pour que l'interaction soit favorisée au maximum. L'autonomie du groupe s'est développée durant cette année. Déjà, la négociation du contrat l'a forcé à s'affirmer comme entité pour faire respecter ses besoins. L'intervenante devenait de plus en plus effacée, mais son support était toujours requis, (Lang, 1972) ; le Club se définissait comme semi-autonome.

\section{Les membres}

Les personnes faisant partie du groupe du courant central sont actives, censées s'engager dans le processus et réaliser ce qu'elles sont capables de faire de façon autonome (Papell et Rothman, 1983). C'était le cas des membres du Club lorsque chacun apportait son support, son expérience propre et assumait la responsabilité de préparer ou de présider une soirée. La contribution au processus de développement ou à la formation des membres, tel ce parent, par exemple, qui enseignait les habiletés d'animation, appartient davantage au modèle à buts sociaux. 


\section{Les activités}

Les activités avaient surtout un caractère éducatif et elles étaient moins passives et rigides que dans le groupe d'éducation structurée. Il y avait réellement de la place pour la spontanéité, l'innovation et la planification (Papell et Rothman, 1983). Mentionnons, entre autres, la soirée où le groupe a assisté à une conférence d'une psychologue scolaire sur le rôle de parent, et la soirée d'improvisation à la Maison des jeunes où des adolescents et adolescentes affrontaient des parents. Les activités de préparation se faisaient individuellement ou en sous-groupes, avec l'intervenante au besoin. Souvent, les gens ne désiraient qu'être rassurés sur leur travail.

\section{Le rôle de l'intervenante}

Ici, l'intervenante a joué un rôle d'enabler pour aider les gens à utiliser leurs capacités; elle l'a particulièrement exercé auprès de la personne identifiée comme leader potentiel, qui sortait d'une expérience de groupe destructrice. Elle a également joué le rôle de facilitateur, et celui d'enseignante à l'occasion. Le rôle de médiateur a été rempli lors de l'établissement du contrat. Cette présence du rôle variable en service social des groupes a été bien soulignée par Henry (1981).

Dans sa phase I, le Club appartenait donc au modèle de réciprocité. L'utilisation du processus de groupe dans l'ici et maintenant n'a pas été exploitée à son maximum, mais le Club a tout de même poursuivi son cheminement. Nous allons maintenant analyser sa deuxième phase.

\section{Le club de parents d'adolescents, phase II : le modèle à buts sociaux}

\section{Le groupe, ses caractéristiques}

La phase II du Club s'est déroulée durant l'année 1984-1985; il restait huit membres. Ces derniers avaient décidé d'orienter leur travail pour faire profiter la communauté de leurs acquis. Ils étaient conscients que d'autres parents avaient les mêmes problèmes et pourraient bénéficier de leur action (Lewis, 1983). Ils ont opté pour l'organisation d'une journée thématique. L'extériorité qui se pressentait à la phase précédente était réellement présente. 
Le programme de la journée portait, d'une part, sur le vécu propre des parents (frustrations, nécessité du dialogue conjugal, etc.) et, d'autre part, sur leur rôle auprès du jeune ; en somme, les thèmes privilégiés du groupe. Le témoignage de deux parents venait relier ces deux thèmes. Le groupe avait opté pour engager et rémunérer des personnes ressources et servir un buffet sur place pour permettre aux gens de rester toute la journée. L'organisation nécessitait donc une recherche de fonds.

À cette phase, le groupe était orienté vers le changement social, non pas pour corriger une injustice mais plutôt pour valoriser le rôle parental dans le milieu. Il visait à augmenter la participation des membres dans leur communauté. Quelques-uns d'entre eux et l'intervenante visaient même le développement d'une ressource permanente pour les parents, soit un groupe d'entraide ou encore une Maison des parents.

On reconnaît le modèle à buts sociaux décrit par Lewis (1983 : 37), où l'on vise la réalisation d'un objectif dans l'environnement social du groupe. Dans ce modèle, le développement du groupe n'est qu'un moyen pour réaliser la tâche et aider les gens à s'épanouir. Ici, il était exclusivement centré sur la tâche. Le vécu parental était devenu secondaire et l'aide mutuelle se manifestait à travers les tâches. Le climat socio-émotif qui régnait était suffisamment chaleureux pour permettre l'exécution efficace des responsabilités de chacun. Cependant, les connaissances théoriques sur l'utilité de ce rôle lui faisant défaut, l'intervenante n'a donc pas fait une utilisation consciente des phénomènes de groupe, ce qui aurait permis aux membres de mieux comprendre ce qui se passait. L'autonomie du groupe était encore plus grande qu'à la phase précédente. II avait une responsable "officielle", répondante pour le Club face à la communauté.

\section{Les membres}

Les membres ne poursuivaient pas, comme tel, des objectifs personnels et n'y adhéraient pas pour trouver du support, comme précédemment. Et, tel que le mentionne Lewis (1983: 39), les objectifs de réalisation personnelle et de croissance se concrétisaient à travers l'exécution des tâches. À titre d'exemple, soulignons le cas d'une mère qui a acquis énormément de confiance en elle, après s'être vu confier la responsabilité de rencontrer un conseiller municipal pour obtenir des fonds pour la journée thématique. Les membres ne constituaient pas une population à risques et se trouvaient en droit de réclamer des services "normaux" pour les parents qui rencontraient des difficultés "normales" lorsque leurs enfants arrivaient à l'adolescence (Lewis, 1983). 


\section{Les activités}

Les activités ont été très diversifiées et axées sur la réalisation de cette journée thématique. Après avoir fixé leurs objectifs, les membres sont passés à l'action. Ils ont: précisé le programme de la journée, recherché des personnes ressources et des sources de financement, composé les communiqués de presse, participé à la publicité télévisée, vu à l'organisation matérielle de la salle et à la préparation du buffet, formulé les mots de bienvenue et de remerciements, élaboré la formule d'évaluation de la journée, et, enfin, sensibilisé les parents de leur environnement à cet événement.

\section{Le rôle de l'intervenante}

L'intervenante a surtout joué un rôle technique de personne ressource auprès du groupe, aidant celui-ci, entre autres, à trouver les ressources et à rédiger les communiqués. Elle a áussi joué un rôle de médiateur pour obtenir des services de secrétariat de la part du C.L.S.C. pour préparer la journée. Les rôles d'animateur et de président étaient remplis par la responsable du groupe auprès de qui l'intervenante a eu un rôle d'enabler afin de la supporter dans ses fonctions.

\section{Conclusion}

Ce groupe unique a connu un cheminement particulier sur une période de plus de trois ans. Comme beaucoup d'expériences pratiques, celle-ci n'adhérait pas en tout point à la théorie. Nous l'avons examinée en détail à partir des différents modèles en service social, en nous attardant au groupe, à ses caractéristiques, aux membres, aux activités, et aux rôles de l'intervenante.

Les personnes qui composaient le groupe ont énormément influencé ce cheminement particulier. En effet, l'intervenante, parallèlement au travail auprès du Club, continuait l'animation de sessions pour parents d'adolescents et, même si elle tentait d'y favoriser l'aide mutuelle ou d'occuper une position moins centrale, aucun groupe n'a émergé de ces sessions. Cela signifie que les personnes qui ont formé le groupe analysé ici étaient beaucoup plus prêtes à un engagement que ce que l'on retrouve habituellement en éducation structurée.

Après cette journée pour les parents d'adolescents du territoire, le groupe s'est dissout, chacun et chacune ayant des projets personnels ou 
communautaires. Cependant, la responsable du groupe et son conjoint ont poursuivi leur action dans la communauté en amorçant, à l'automne 1985, la mise sur pied d'un groupe d'entraide.

\section{Note}

1 Le Bureau de consultation jeunesse inc., organisme privé sans but lucratif, a plusieurs points de services à travers le grand Montréal. Depuis plus de quinze ans, il s'est démarqué par son dévouement à la cause des jeunes en difficlté et par le caractère innovateur de son action.

\section{Références bibliographiques}

Darveau-Fournier, L. et A. Home, "La spécificité du service social des groupes", Service social, vol. 29, nos 1-2, $1980: 16-31$.

DARVeau-Fournier, L. et A. HOME, "Les groupes de service social à Québec: recherche des rapports entre théorie et pratique", Service social, vol. 32, nos 1-2, 1983 : 129-155.

DRUM, D.J. et J.E. KNOTT, Structured Groups for Facilitating Development, New York, Human Sciences Press, 1977, 284p.

Germain, C. et A. Gitterman, The Life Model of Social Work Practice, New York, Columbia University Press, 1980, 376p.

HeNRY, S., Group Skills in Social Work : A Four Dimensional Approach, chap 2, Itasca, Peacock Publishers, 1981. (Traduction synthèse de A. Home, Université Laval, 1985.)

LANG, N., "A broad-range model of practice in the social work group ", Social Service Review, vol. 46, no 1, 1972: 76-82. (Traduction synthèse de G. Fortier et A. Home, parue dans le recueil de textes pour les cours SVS-62534 et SVS63086, Université Laval, 1984.)

LEWIS, E., "Le service social des groupes dans la vie communautaire : caractéristiques des groupes et du rôle du travailleur social ", Service social, vol. 32, nos 1-2, 1983 : 32-49.

PAPELL, C. et B. RothMAN, "Social group work models : possession and heritage", Journal of Education for Social Work, vol. 2, no 2, 1966 : 66-77.

PAPELl, C. et B. Rothman, "Le modèle du courant central du service social des groupes en parallèle avec la psychothérapie et l'approche de groupe structuré ", Service social, vol. 32, nos 1-2, 1983 : 11-31.

SHULMAN, L., The Skills of Helping Individuals and Groups, $2^{\mathrm{e}}$ édition, Itasca (III.), Peacock, 1984, 432p. 